Check for updates

Cite this: RSC Adv., 2018, 8, 36043

\title{
Friction-induced selective etching on silicon by TMAH solution
}

\author{
Chao Zhou, Jiaming Li, Lei Wu, Guangran Guo, Hongbo Wang, (DD Peng Chen, \\ Bingjun Yu (D) and Linmao Qian (D)
}

Friction-induced selective etching provides a new thought direction in the field of nanotechnology with high resolution, low cost, flexibility and site control. In this work, it was found that the scratched area on a silicon surface can play a role as a mask against etching in tetramethyl ammonium hydroxide (TMAH) solution, resulting in the formation of protrusive hillocks. Friction-induced selective etching was found to depend on the temperature and etching time. The hillock height initially increased with the temperature or etching time, and then the hillock disappeared due to the mask etching off. In contrast, the applied normal load for scratching on silicon had little effect on the hillock height produced by selective etching in TMAH solution. Further analysis showed that crystal distortions or crystal amorphization could act as a mask against selective etching on silicon. Through control tip traces for scratching, different patterns can be produced on the silicon surface by friction-induced selective etching in TMAH solution. These results can enrich the fundamental aspects of scanning probe microscope (SPM)-based nanolithography, and provide an alternative method to produce nanostructures for various applications.

Received 23rd August 2018

Accepted 12th October 2018

DOI: $10.1039 / \mathrm{c} 8 \mathrm{ra07064d}$

rsc.li/rsc-advances pyrocatechol (EDP). ${ }^{7-9}$ However, during the etching process, $\mathrm{KOH}$ can introduce metal ions onto the fabrication surface, ${ }^{\mathbf{1 0 , 1 1}}$ while EDP is highly toxic and is not conducive to experimental operation. Compared with other etching agents, TMAH is stable and does not decompose at temperatures below $130^{\circ} \mathrm{C}$. Thus, it is a commonly used etching agent in the MEMS process. ${ }^{12}$ The low etching rate of TMAH makes the fabrication process controllable. Furthermore, TMAH is low toxic compared to EDP,$^{12}$ and can be easily removed after etching.

In recent years, friction-induced selective etching has become a popular method for fabricating surface nanostructures without any masks, due to the simple process, low cost and flexibility, as well as the potential for nondestructive nanofabrication. ${ }^{\mathbf{1 3 , 1 4}}$ Nanofabrication by friction-induced selective etching can be applied to a wide variety of materials, such as silicon, gallium arsenide, quartz, glass and so on. ${ }^{15-18}$ Due to the small load applied on a sharp tip for scratching, frictioninduced selective etching can greatly extend the working life of the tip. Also, high aspect-ratio nanostructures can be produced on monocrystalline silicon through friction-induced selective etching with $\mathrm{Si}_{3} \mathrm{~N}_{4}$ masks. ${ }^{19}$ The friction-induced selective etching method opens up new avenues for producing templates for nanoimprint lithography on silicon and quartz and can be used to fabricate patterned nanochannels in micro/ nano fluidic systems for drug delivery, ion transporters, DNA translocators, etc. It also provides a new approach to modify surface hydrophobicity or tribology properties. However, the friction-induced selective etching of silicon has been
Tribology Research Institute, Key Laboratory of Advanced Technologies of Materials (Ministry of Education), Southwest Jiaotong University, Chengdu 610031, Sichuan Province, P. R. China. E-mail: bingjun@swjtu.edu.cn; Fax: +8628 87603142; Tel: $+862887634181$ 
demonstrated using only $\mathrm{KOH}$ solution until now. ${ }^{\mathbf{1 8 2 0}}$ It is still not understood whether TMAH has a selective etching effect.

In this work, performances of friction-induced selective etching in TMAH solution were investigated involving the effects of etching temperature, etching time and scratching load. The mechanism involved in friction-induced selective etching of silicon was also evaluated based on contrastive tests. The fabrication of various surface patterns were demonstrated with controlling tip traces for scratching.

\section{Results and discussion}

\section{Effects of etching time and temperature on selective etching}

When the diamond tip scratches over silicon surface, lattice deformation occurs on the scratched area, forming amorphous layers and distortion structures, as well as silicon oxides. ${ }^{21}$ The scratched area can act as a mask against TMAH etching, and protrusive hillocks or nanostructures can be produced from the scratched trace because the etching rate of crystal silicon is much higher than that of the scratched area. Fig. 1 illustrates the effect of etching temperature on the formation of nanostructures originating from scratches produced under applied load of $10 \mu \mathrm{N}$. Generally, etching for $30 \mathrm{~s}$ at different temperatures led to the formation of hillocks. When the temperature was $25{ }^{\circ} \mathrm{C}$, selective etching resulted in the formation of protrusive hillocks within $15 \mathrm{~min}$, while selective etching was not observed after etching for $5 \mathrm{~min}$ at $35{ }^{\circ} \mathrm{C}$ and above. It was found that higher temperature led to faster etching for the same etching duration.

The hillock height was plotted as a function of etching time after etching at different temperatures, as shown in Fig. 2. It was found that the height increased as the temperature rose to $50{ }^{\circ} \mathrm{C}$ within $1 \mathrm{~min}$. Due to the rapid chemical reaction between
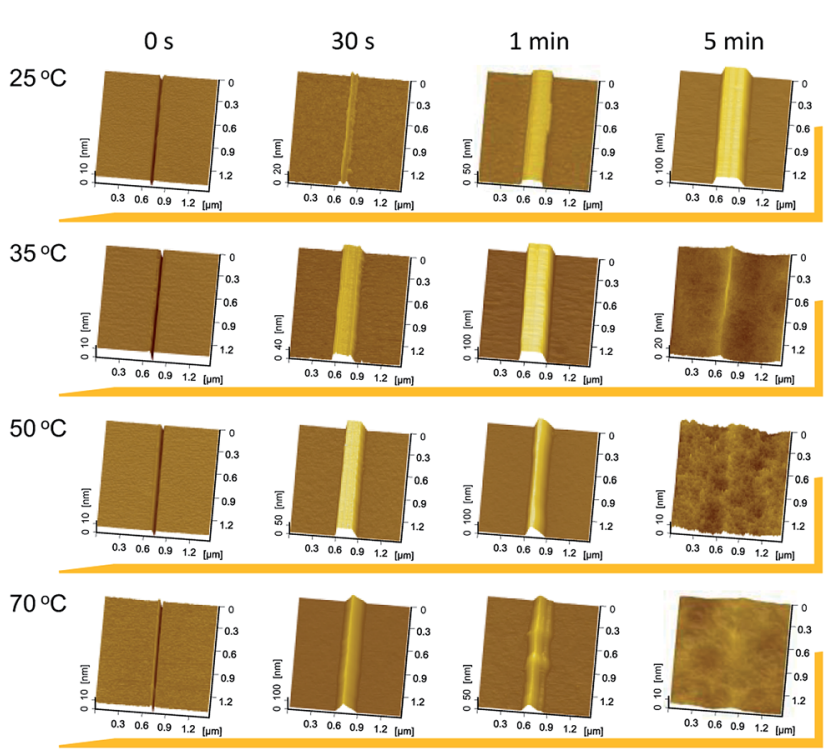

Fig. 1 AFM images of the hillocks produced by friction-induced selective etching in TMAH solution at different temperatures and etching times. Here time $0 \mathrm{~s}$ refers to the silicon wafer with scratches before the etching.

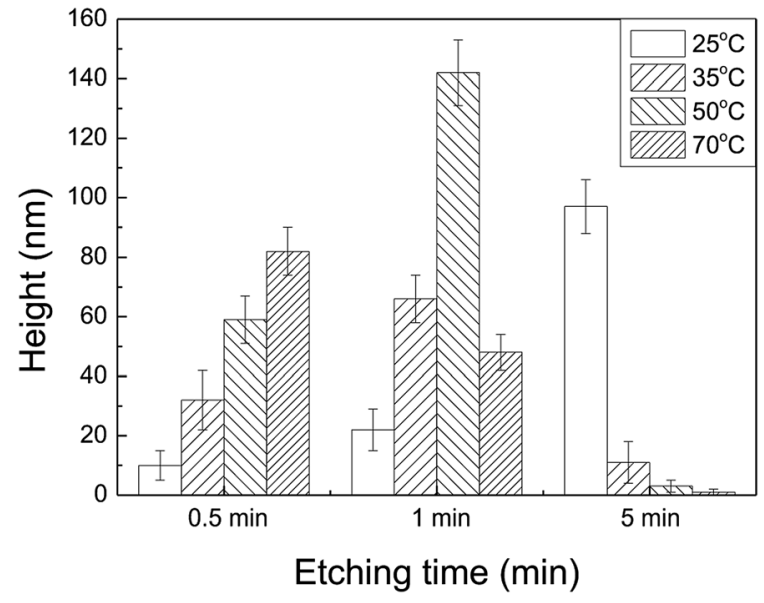

Fig. 2 Variation of the hillock height with the etching time after etching at different temperatures.

silicon oxide and TMAH at high temperature, 5 min of etching caused the collapse of the hillock, and selective etching was not observed at temperatures above $50{ }^{\circ} \mathrm{C}$. Consequently, the selective etching lasted for a longer time, even though low temperature led to relatively low etching rate. Therefore, the results indicate that low temperature, i.e., $25{ }^{\circ} \mathrm{C}$, facilitated controllable selective etching.

Based on the results in Fig. 1 and 2, it is clear that the etching time had a significant impact on the height of protrusive hillocks on silicon surface by selective etching in TMAH solution. The etching time-dependent height of the hillocks was further investigated at $25{ }^{\circ} \mathrm{C}$. As shown in Fig. 3, the hillock height increased rapidly as the etching time increased from 0 to $8 \mathrm{~min}$, and the maximum height reached $136 \mathrm{~nm}$. However, the hillock height decreased gradually to $0 \mathrm{~nm}$ after $8 \mathrm{~min}$. It is expect that the top materials of the newly formed hillock, which is at the junction of inclined $\mathrm{Si}(111)$ planes on both sides of the hillock, have high chemical activity, and the hillock top would be etched off rapidly, leading to the disappearance of the hillock after long-time etching. Therefore, $8 \mathrm{~min}$ was used as the optimum etching time for nanofabrication in the present study.

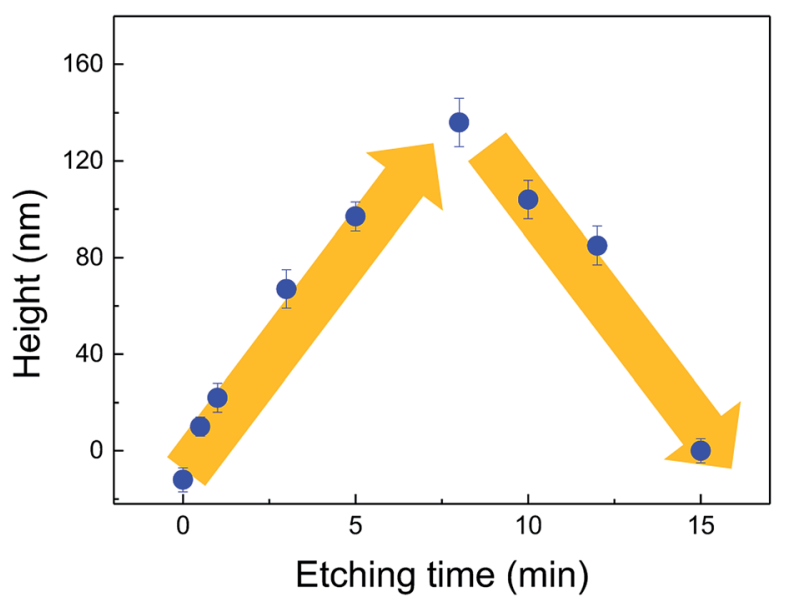

Fig. 3 Variation of hillock height with etching time after etching at $25^{\circ} \mathrm{C}$. 


\section{Effect of scratching load on selective etching}

The effect of applied normal load for scratching on the formation of hillock by selective etching was further investigated. With a diamond tip $(R=2 \mu \mathrm{m})$, a scratch was produced under continuously linear applied normal load from 0 to $6 \mathrm{mN}$. It was observed that, with the increase in applied load, the scratch evolved from a protrusive hillock to a $13 \mathrm{~nm}$-groove at $6 \mathrm{mN}$. As shown in Fig. 4(a), the transition load was $2 \mathrm{mN}$, corresponding to a maximum Hertz contact pressure of 11.5 GPa. ${ }^{22,23}$ Fig. 4(b) demonstrates the formation of surface nanostructures after etching in TMAH solution for up to $8 \mathrm{~min}$. After etching for 1 to $5 \mathrm{~min}$, the entire scratch consisting of both hillock and groove changed into a high hillock, and no obvious change was observed in the transition area of the scratch. However, due to the limited deformation of the slight hillock-shaped scratch created under low load, the tip-affected mask disappeared and almost no hillocks were produced by the selective etching for $8 \mathrm{~min}$. In contrast, an obvious protrusive hillock was formed from the scratch area under higher normal load, suggesting that a thicker mask can facilitate long-time etching. In addition, with the increase in load from $2 \mathrm{mN}$ to $6 \mathrm{mN}$, the top width of the protrusive hillock increased from $827 \mathrm{~nm}$ to $1702 \mathrm{~nm}$, while the hillock remained the same height.

The increase in the top width can be ascribed to the change in contact area during tip scratching. The contact area between the tip and silicon surface increased with the applied normal load, and the top width changed in a similar manner. Moreover, the hillock height was controlled by the mask of deformed layer arising from tip scratching. Therefore, the hillock maintained the same height and was not dependent on the applied load (from $\mu \mathrm{N}$ to $\mathrm{mN}$ level) as long as the mask was active.

\section{Etching mechanism}

Since the etching rate of crystal silicon is much higher than that of silicon oxide, the oxide layer can act as a mask against etching. ${ }^{24}$ However, much remains unknown regarding the role of crystal distortions and amorphous structures underneath the scratch during selective etching by TMAH solution. To further study the mechanism, groove-shaped scratches and protrusive hillocks were produced on silicon surface by tip scratching and then used for the selective etching. For comparison, silicon oxide structure, which was produced by local anodic oxidation (LAO) on AFM, was also etched by TMAH solution. During the LAO process, the silicon oxide can be generated when a bias voltage is applied to silicon surface with absorbed water. ${ }^{25}$

The scratching of a diamond tip on silicon surface can result in lattice distortions containing surface oxides on top and deformation layer beneath, such as amorphous and crystal distortions. ${ }^{25}$ However, the effect of the deformation layer on the selective etching process is still unclear. The performances of different tip-affected structures against selective etching are shown in Fig. 5. As a reference, the LAO structure fabricated by conductive AFM was used for the selective etching in TMAH, as shown in Fig. 5(a). The images in Fig. 5(b)-(d) show the formation of protrusive hillocks by selective etching with $\mathrm{HF}$ and TMAH solutions in turn, where HF solution was used for removing silicon oxides on top. In Fig. 5(b), a protrusive hillock was produced from the mechanical scratch after two-step etching in HF and TMAH solutions, and it can be deduced that the deformation layer beneath the groove resisted TMAH etching. Similarly, friction-induced hillocks produced at different sliding velocities evolved into higher hillocks after being etched in turn by HF and TMAH solutions, as shown in

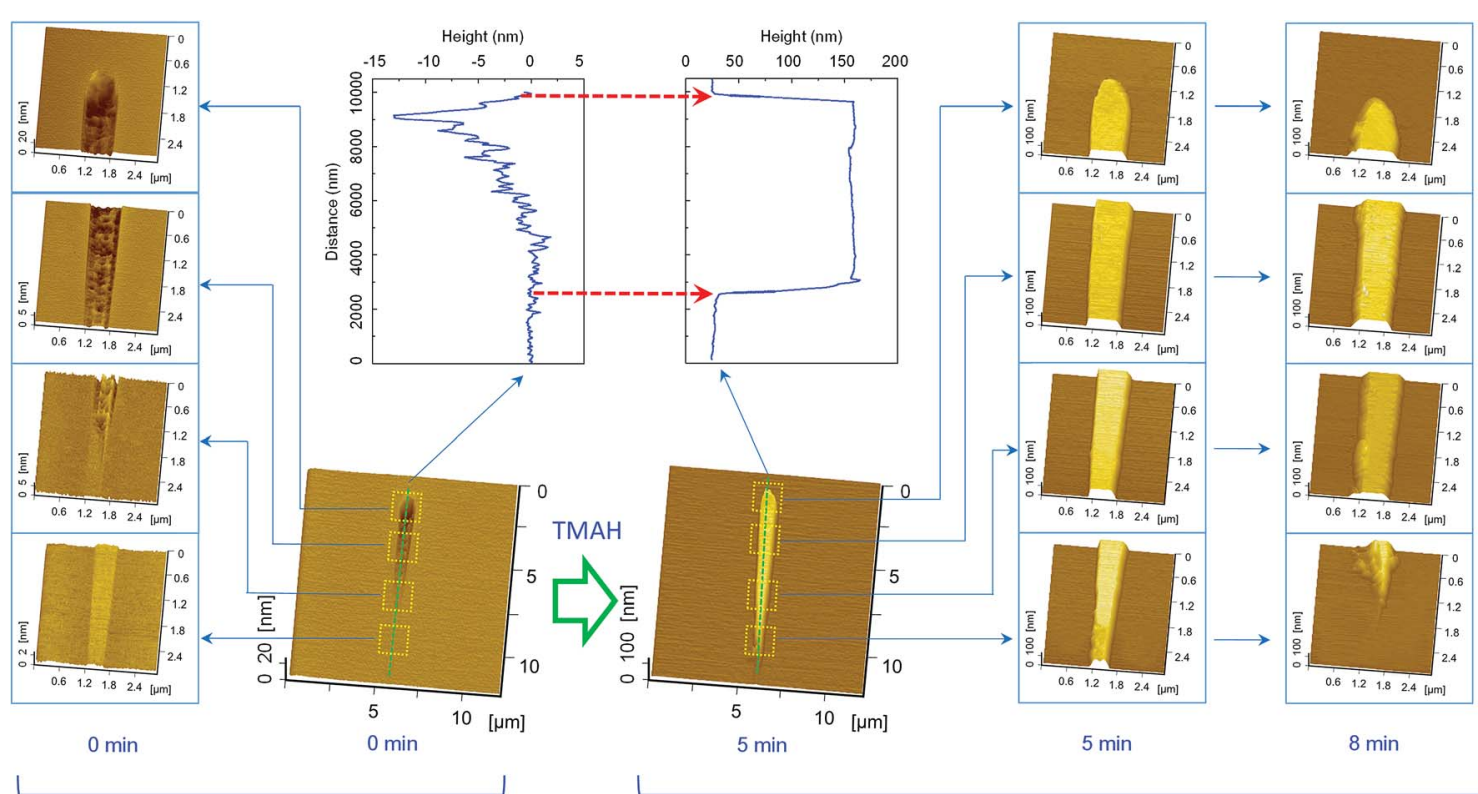

(a)

(b)

Fig. 4 Effect of scratching load on selective etching by TMAH solution. (a) AFM images and cross-section profile of a scratch produced by a diamond tip $(R=2 \mu \mathrm{m})$ under continuously linear applied normal load from 0 to $6 \mathrm{mN}$. (b) AFM images and cross-section profile of the fabricated hillock after being etched for 5 to $8 \mathrm{~min}$. The zoomed images show the details of (a) the scratch or (b) hillock corresponding to different applied load for scratching. The temperature was $25^{\circ} \mathrm{C}$. 
(a)
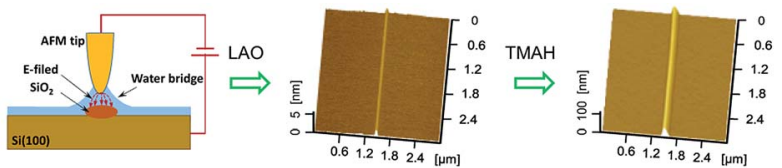

(b)
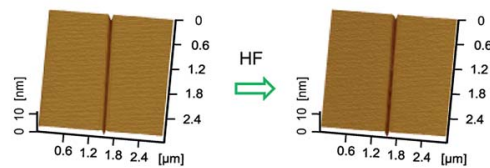

$$
\stackrel{\text { TMAH }}{\Rightarrow}
$$

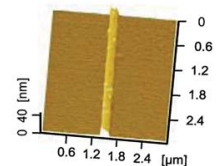

(c)
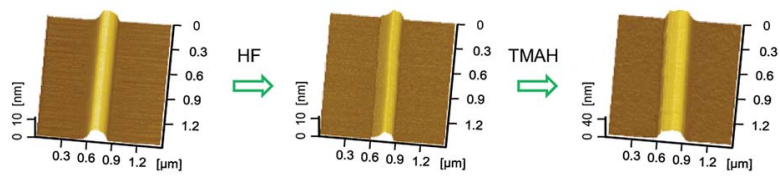

(d)
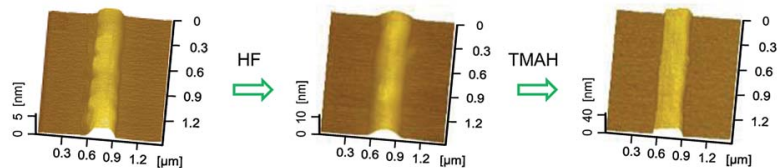

Fig. 5 AFM images of the structures produced by friction-induced selective etching with different masks. (a) AFM images of the LAO mask before and after etching. The LAO mask was produced by a conductive tip at bias voltage of $7 \mathrm{~V}$. (b) AFM images of a groove before and after etching by HF and TMAH solutions. The scratch was firstly dipped with HF solution to remove surface oxides, and then etched in TMAH solution. (c) AFM images of a protrusive hillock fabricated by selective etching at sliding speed of $10 \mu \mathrm{m} \mathrm{s}^{-1}$ (d) AFM images of a protrusive hillock fabricated by selective etching at sliding speed of $1000 \mu \mathrm{m} \mathrm{s}^{-1}$, where the wider hillock can be ascribed to the vibration in AFM scanning. In this figure, the etching time was 5 min for HF solution, and 1 min for TMAH solution, respectively. The contact pressure for the hillock formation was $5.8 \mathrm{GPa}$, and the number of scratching cycles was 100 (Fig. 5(c) and (d)).

Fig. 5(c) and (d). The hillock height changed from $7 \mathrm{~nm}$ (Fig. 5(c)) or $3 \mathrm{~nm}$ (Fig. 5(d)) to more than $30 \mathrm{~nm}$ after being etched in TMAH for $1 \mathrm{~min}$. It was found that the frictioninduced hillock on silicon created at low velocity of $10 \mu \mathrm{m} \mathrm{s}^{-1}$ (Fig. 5(c)) was mainly amorphous, such as dislocations and stacking faults, while the silicon hillock created at high sliding velocity of $1000 \mu \mathrm{m} \mathrm{s}^{-1}$ (Fig. 5(d)) was constructed by crystal distortions. ${ }^{26}$ The scratch-induced silicon crystal distortion consists of amorphous layer and deformed lattices, such as slip lines and stacking faults, ${ }^{26,27}$ which is structurally different from the oxides mask. During the anisotropic etching of silicon surface, the distortions beneath the scratched area can prevent the attack from etching solution, and present a low etching rate. In contrast, the chemical inertness of silicon oxide results in a low etching rate in TMAH solution. Therefore, crystal distortions and amorphous silicon also resisted TMAH etching.

For comparison, friction-induced selective etching in TMAH solution was also conducted on $\mathrm{Si}(111)$ and $\mathrm{Si}(110)$ surfaces. Similar hillocks were produced on $\mathrm{Si}(110)$ surface by etching in TMAH solution (Fig. 6), while no obvious selective etching was observed on $\mathrm{Si}(111)$. This can be ascribed to the fact that (111) crystal plane has a very low etching rate, which is comparable to the crystal deformation mask created by scratching. ${ }^{28}$ During the etching, (111) plane played a role in preventing the etching, resulting in an inclined plane with an angle of $54.74^{\circ}$. (a)

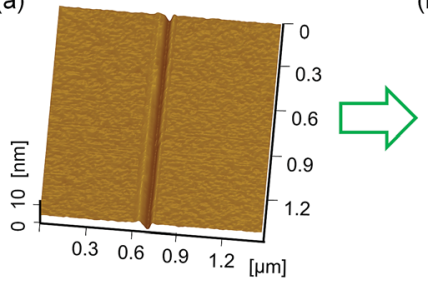

(b)

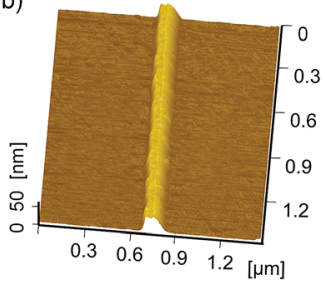

Fig. 6 AFM image of (a) a scratch on Si(110) and (b) a hillock produced by selective etching in TMAH for $1 \mathrm{~min}$.

\section{Nanopatterning of Si surface by selective etching in TMAH solution}

Friction-induced selective etching is a low-cost and flexible approach for surface patterning and fabrication. It should be noted that scanning probe microscope (SPM)-based nanofabrication by direct scratching requires a high normal load or pressure, which can cause the tip to be easily worn out, even a diamond tip. In contrast, selective etching was realized as long as the oxidation or crystal deformation occurred during scratching, and the applied normal load for scratching had minimal effect on the fabrication (Fig. 4). In other words, the fabrication by friction-induced selective etching caused less wear to the tip employed. Also, the etching with TMAH can result in high-quality surface. As shown in Fig. 7, after the same etching duration, the silicon surface after etching with TMAH solution is much smoother than that after etching with $\mathrm{KOH}$. Fig. 8 presents different patterns produced on $\mathrm{Si}(100)$ by the friction-induced selective etching in TMAH solution, which would be helpful to produce shape-controlled patterns for optical substrates and microchannels. ${ }^{29-32}$

When a traditional SPM scanning is employed for surface lithography, SPM tip could be easily worn due to high contact pressure for plastic deformation of substrate material during scratching. ${ }^{32}$ In contrast, high hillock or deep channels can be fabricated by friction-induced selective etching, and the process is realized at low applied load for scratching, which reduces the wear of SPM tip. It is also noted that the hillock height resulting from friction-induced selective etching is almost independent of applied load for scratching (Fig. 4), which can avoid the effect of applied load difference on fabrication results. On the other hand, any 2-dimentional surface patterns can be produced on silicon surface at low cost based on the flexibility of SPM in scanning, and it is meaningful for designing tiny channels for nanofluidics, site-controlled patterns for molecular beam epitaxy (MBE) formation of high-quality optic structures, and
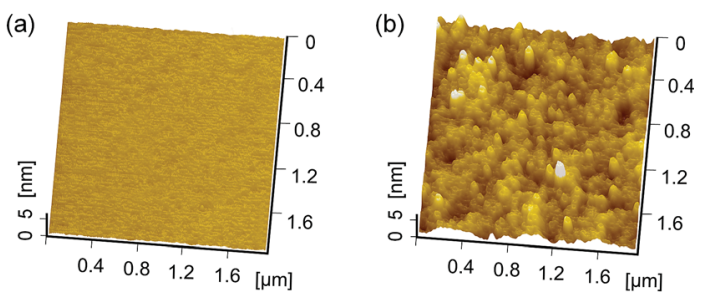

Fig. 7 AFM images and the corresponding profiles of Si(100) surface etched by (a) TMAH and (b) $\mathrm{KOH}$ solutions for 5 min at $25^{\circ} \mathrm{C}$. 


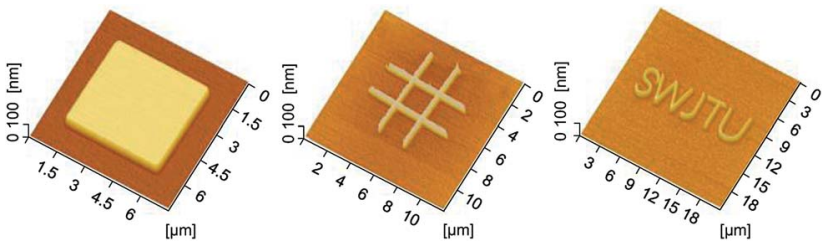

Fig. 8 Nanopatterning of Si by friction-induced selective etching.

some functional patterns for adherent cell or bacterial analysis. ${ }^{29,30,33,34}$ As a result, the proposed study can enrich the fundamental aspects of SPM-based nanolithography, and provides an alternative method to produce nanostructures for various applications.

\section{Experimental}

\section{Materials}

P-type Si wafers, doped with boron, were purchased from MEMC Electronic Materials, Inc., USA. The surface root-meansquare (RMS) roughness was measured as about $0.10 \mathrm{~nm}$ over an area of $2 \mu \mathrm{m} \times 2 \mu \mathrm{m}$ by an atomic force microscope (AFM; SPI3800N, Seiko Instruments Inc., Tokyo, Japan). Commercially available TMAH solution ( $25 \mathrm{wt} \%$ in water, Sinopharm Group Chemical Reagent Co., Ltd., China) was used for the etching. Before fabrication, $\mathrm{Si}$ wafer was cut into square shapes with a size of $\sim 1 \mathrm{~cm} \times 1 \mathrm{~cm}$. Then, the samples were dipped in $5 \mathrm{wt} \% \mathrm{HF}$ solution for $5 \mathrm{~min}$ to etch away the native Si oxide layer for further tests. ${ }^{35}$

\section{Fabrication}

The fabrication process consisted of two steps: scratching and post-etching in TMAH. The scratching was conducted by a diamond tip with a radius $R$ of $20 \mathrm{~nm}$ under an applied normal load of $10 \mu \mathrm{N}$ on AFM. The depth of these nanoscratches was 5 to $10 \mathrm{~nm}$. With a diamond tip $(R=2 \mu \mathrm{m})$, nanoscratches were produced under continuously linear applied normal load from 0 to $6 \mathrm{mN}$ on a scratcher (Ti-900, Hysitron Inc., USA). Then, the scratched $\mathrm{Si}$ wafers were etched in TMAH solution under specified conditions, where the temperature was controlled by a water bath thermostat with an accuracy of $\pm 0.5^{\circ} \mathrm{C}$. Then the samples were ultrasonically washed with ethanol for $3 \mathrm{~min}$, rinsed with deionized water for $3 \mathrm{~min}$ and dried with dry nitrogen flow in turn. Finally, the images of fabricated nanostructures were acquired in situ from the scratched area by AFM scanning with a $\mathrm{Si}_{3} \mathrm{~N}_{4}$ tip $(R \approx 20 \mu \mathrm{m})$. For comparison, the selective etching of protrusive hillocks on silicon, produced by a diamond tip $(R=500 \mu \mathrm{m})$ on AFM, was also investigated for evaluating the etching mechanism in TMAH solution.

\section{Conclusions}

In this work, the friction-induced selective etching of silicon surface in TMAH solution was investigated for fabricating nanostructures. The main conclusions of this study are summarized as follows.
(1) The mechanically scratched area on silicon surface can function as a mask against etching in TMAH solution.

(2) The hillock formation by friction-induced selective etching was found to depend on the temperature and etching time. The selective etching rate increased with the temperature, while selective etching disappeared with the increase in etching time. In contrast, the applied normal load for scratching on silicon had minimal effect on the hillock height produced by selective etching in TMAH solution.

(3) In addition to silicon oxide, crystal distortions and amorphous silicon also resisted TMAH etching. Based on the proposed mechanism, a low-cost and flexible approach for patterning and fabrication of silicon surface can be realized by friction-induced selective etching.

\section{Conflicts of interest}

There are no conflicts to declare.

\section{Acknowledgements}

The authors express their thanks for the financial support provided by the Sichuan Science and Technology Program (2018HH0151) and the National Natural Science Foundation of China (51775462).

\section{Notes and references}

1 K. Prashanthi, M. Mandal, S. P. Duttagupta, R. Pinto and V. R. Palkar, Sens. Actuators, A, 2011, 166(1), 83-87.

2 M. A. Hopcroft, W. D. Nix and T. W. Kenny, J. Microelectromech. Syst., 2010, 19(2), 229-238.

3 S. Dutta, M. Imran, P. Kumar, R. Pal, P. Datta and R. Chatterjee, Microsyst. Technol., 2011, 17(10-11), 16211628.

4 P. Pal and K. Sato, Microsyst. Technol., 2010, 16(7), 11651174.

5 I. W. Rangelow, J. Vac. Sci. Technol., A, 2003, 21(4), 15501562.

6 M. J. De Boer, J. G. E. Gardeniers, H. V. Jansen, E. Smulders, M. J. Gilde, G. Roelofs, J. N. Sasserath and M. Elwenspoek, J. Microelectromech. Syst., 2002, 11(4), 385-401.

7 C. N. Jin, B. J. Yu, C. Xiao, L. Chen and L. M. Qian, Nanoscale Res. Lett., 2016, 11, 229.

8 V. Swarnalatha, A. V. Narasimha Rao, A. Ashok, S. S. Singh and P. Pal, J. Micromech. Microeng., 2017, 27, 085003.

9 R. Iosub, C. Moldovan and M. Modreanu, Sens. Actuators, A, 2002, 99(1-2), 104-111.

10 Y. Zhang, Y. Q. Zhou, Z. Y. Jiang, F. Z. Liu and M. F. Zhu, Phys. Status Solidi C, 2010, 7(3-4), 1025-1028.

11 M. Shikida, K. Sato, K. Tokoro and D. Uchikawa, Sens. Actuators, A, 2000, 80(2), 179-188.

12 G. Z. Yan, P. C. H. Chan, I. M. Hsing, R. K. Sharma, J. K. O. Sin and Y. Y. Wang, Sens. Actuators, A, 2001, 89(12), 135-141.

13 J. Guo, C. Xiao, B. Peng and L. M. Qian, $R S C A d v .$, 2015, 5, 100769-100774. 
14 J. Guo, B. J. Yu, L. Chen and L. M. Qian, Sci. Rep., 2015, 5, 16472.

15 J. Guo, C. F. Song, X. Y. Li, B. J. Yu, H. S. Dong, L. M. Qian and Z. R. Zhou, Nanoscale Res. Lett., 2012, 7, 152.

16 C. F. Song, B. J. Yu and L. M. Qian, Micro Nano Lett., 2013, 8(10), 735-739.

17 C. F. Song, X. Y. Li, S. X. Cui, H. S. Dong, B. J. Yu and L. M. Qian, Nanoscale Res. Lett., 2013, 8, 140.

18 C. F. Song, B. J. Yu, M. Wang and L. M. Qian, $R S C A d v .$, 2015, 5(97), 79964-79968.

19 J. Guo, B. J. Yu, X. D. Wang and L. M. Qian, Nanoscale Res. Lett., 2014, 9, 241.

20 P. Tang, B. J. Yu, J. Guo, C. F. Song and L. M. Qian, Nanoscale Res. Lett., 2014, 9, 59.

21 B. J. Yu, H. S. Dong, L. M. Qian, Y. F. Chen, J. X. Yu and Z. R. Zhou, Nanotechnology, 2009, 20, 465303.

22 B. J. Yu, H. T. He, L. Chen and L. M. Qian, Wear, 2017, 374, 29-35.

23 B. J. Yu, L. M. Qian, H. S. Dong, J. X. Yu and Z. R. Zhou, Wear, 2010, 268(9-10), 1095-1102.

24 J. S. You, D. Kim, J. Y. Huh, H. J. Park, J. J. Pak and C. S. Kang, Sol. Energy Mater. Sol. Cells, 2001, 66(1-4), 37-44.

25 D. Stiévenard and B. Legrand, Prog. Surf. Sci., 2006, 81, 112140.
26 B. J. Yu, X. Y. Li, H. S. Dong, Y. F. Chen, L. M. Qian and Z. R. Zhou, J. Phys. D: Appl. Phys., 2012, 45, 145301.

27 L. Chen, J. L. Wen, P. Zhang, B. J. Yu, C. Chen, T. B. Ma, X. C. Lu, S. H. Kim and L. M. Qian, Nat. Commun., 2018, 9, 1542.

28 C. N. Jin, B. J. Yu, X. X. Liu, C. Xiao, H. B. Wang, S. L. Jiang, J. Wu, H. Y. Liu and L. M. Qian, Appl. Surf. Sci., 2017, 425, 227-232.

29 W. sparreboom, d. B. A. Van and J. C. eijkel, Nat. Nanotechnol., 2009, 4, 713.

30 X. Y. Chen and L. Zhang, Sens. Actuators, B, 2017, 254, 648659.

31 A. Krost and A. Dadgar, Mater. Sci. Eng., B, 2002, 93(1-3), 7784.

32 A. G. Khurshudov, K. Kato and H. Koide, Tribol. Lett., 1996, 2(4), 345-354.

33 J. Norman, M. J. Kennedy, J. Selvidge, Q. Li, Y. Wan, A. Y. Liu, P. G. Callahan, M. P. Echlin, T. M. Pollock, K. M. Lau, A. C. Gossard and J. E. Bowers, Opt. Express, 2017, 25(4), 3927-3934.

34 C. Berne, C. K. Ellison, A. Ducret and Y. V. Brun, Nat. Rev. Microbiol., 2018, 10(16), 616-627.

35 G. R. Guo, J. M. Li, C. B. Deng, Q. P. He, Q. Y. Yu, C. Zhou, X. X. Liu, S. L. Jiang, L. Chen, B. Y. Yu and L. M. Qian, Appl. Phys. Express, 2018, 11, 116501. 